\title{
Ultrasonographic Findings in the Diagnosis of Obstructed Right Hemivagina and Ipsilateral Renal Agenesis (OHVIRA) Syndrome Associated with Uterus Didelphys with MRI Correlation - An Unusual Cause of Cyclic Abdominal Pain in a Prepubertal Girl - A Case Report
}

\author{
Yash Patel ${ }^{1}$, Ishrat Fatima², Shivani Mahendru³ ${ }^{3}$ Kritika Agrawal ${ }^{4}$, Aastha Garg 5
}

1, 3, 4, 5 Department of Radiodiagnosis, Maharishi Markandeshwar Institute of Medical Sciences and Research, Maharishi

Markandeshwar (Deemed to Be University) Mullana, Ambala, Haryana, India. ${ }^{2}$ Department of Physiotherapy \&

Rehabilitation, Maharishi Markandeshwar (Deemed to Be University) Mullana, Ambala, Haryana, India.

\section{INTRODUCTION}

Herlyn-Werner-Wunderlich syndrome, also known as Obstructed Hemivagina and Ipsilateral Renal Anomaly (OHVIRA) syndrome, is a congenital anomaly in which unilateral Mullerian duct anomaly is associated with ipsilateral renal anomaly, mainly renal agenesis. OHVIRA accounts 0.16 - $10 \%$ of Mullerian duct anomalies while Mullerian duct anomalies have prevalence of $2-3 \%$. It is an unusual cause of cyclic abdominal pain during menstruation in young females. ${ }^{1}$ This syndrome causing obstructive symptoms after menarche but remains undiagnosed during infancy or childhood. At times this syndrome may be identified after the assessment and evaluation of a patient with repeated pregnancy loss or infertility. Clinical diagnosis is very demanding and requires imaging studies in which ultrasound and MRI (Magnetic Resonance Imaging) play an important role in the classification, identification, diagnosis and treatment scheme plan. Magnetic Resonance Imaging is beneficial in characterising the vaginal septum and didelphic uterus for pre-operative planning. Considering the clinical presentation in association with the imaging findings, is condemnatory for quick diagnosis in an endeavour to obviate problems and complications such as adhesions or endometriosis from infections and infertility. ${ }^{2}$ Awareness of this condition can help in detecting this condition on sonography. MRI can provide exquisite detail of the anomaly and its extent.

\section{PRESENTATION OF CASE}

A 13 year aged female patient who had recent onset of menarche presented with cyclical abdominal pain during menstruation with tenderness in the right lower abdomen. Laboratory investigations were within standard limits. External genitalia also appear normal. Ultrasonographic examination was requested for this patient which revealed two uterine horns \& collection with internal echoes in the region of right sided cervix and right hemivagina [Figure 1] a, [Figure 2] a. which is extending up to lower endometrial cavity on same side. [Figure 3] a. Based on clinical and sonographic findings, possibility of uterus didelphys with obstructed hemivagina was kept in mind. Furthermore, there was no visualization of the kidney \& ureter on right side [Figure 4] a. Then MRI was done for confirmation of the diagnosis [Figure 1] b, [Figure 2] b, [Figure 3] b and [Figure 4] b. There was also associated haematosalpinx on right side without any evidence of adnexal mass. [Figure 5] [Figure 6] A diagnostic laparoscopy was carried out to determine the area of haemorrhagic collection and to evaluate the adnexa for any endometriosis emerging from retro-grade flow of menstruation. The patient underwent the resection of obstructive transverse vaginal septum with drainage of the dark brown-coloured chronic haematoma with complete evacuation of vaginal collection and relief of symptoms.
Corresponding Author:

Dr. Yash Patel,

5, Apurva Bunglows,

B/H Saheli Party Plot,

Near Bhuyangdev Cross Road,

Memnagar, Ahmedabad - 380052,

Gujarat, India.

E-mail: yashpatel2897@gmail.com

DOI: $10.14260 /$ jemds/2020/748

How to Cite This Article:

Patel Y, Fatima I, Mahendru S, et al. Ultrasonographic findings in the diagnosis of obstructed right hemivagina and ipsilateral renal agenesis (OHVIRA) syndrome associated with uterus didelphys with MRI correlation- an unusual cause of cyclic abdominal pain in a prepubertal girl - a case report. J Evolution Med Dent Sci 2020;9(45):3408-3410, $10.14260 / \mathrm{jemds} / 2020 / 748$ DOI:

Submission 04-08-2020,

Peer Review 28-09-2020,

Acceptance 05-10-2020,

Published 09-11-2020.

Copyright (C) 2020 Yash Patel et al. This is an open access article distributed under Creative Commons Attribution License [Attribution 4.0 International (CC BY 4.0)] 


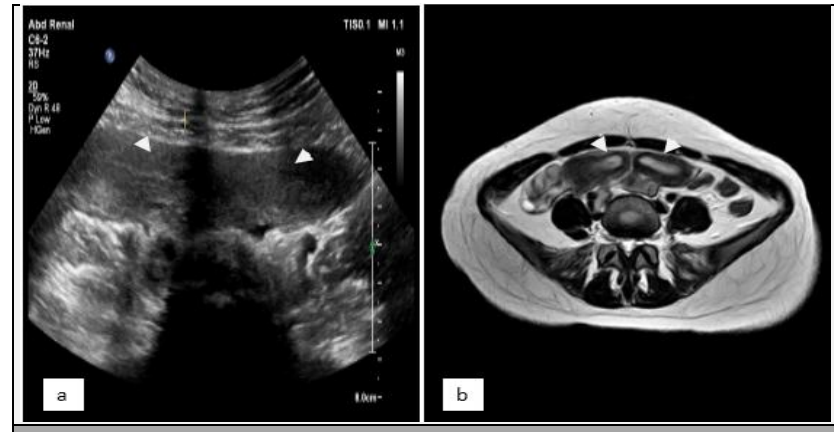

Figure 1. Axial Ultrasound (a) and Axial Magnetic Resonance Imaging T2 Weighted (b) Showing Two Uterine Horns (Arrows) - Uterine Didelphys
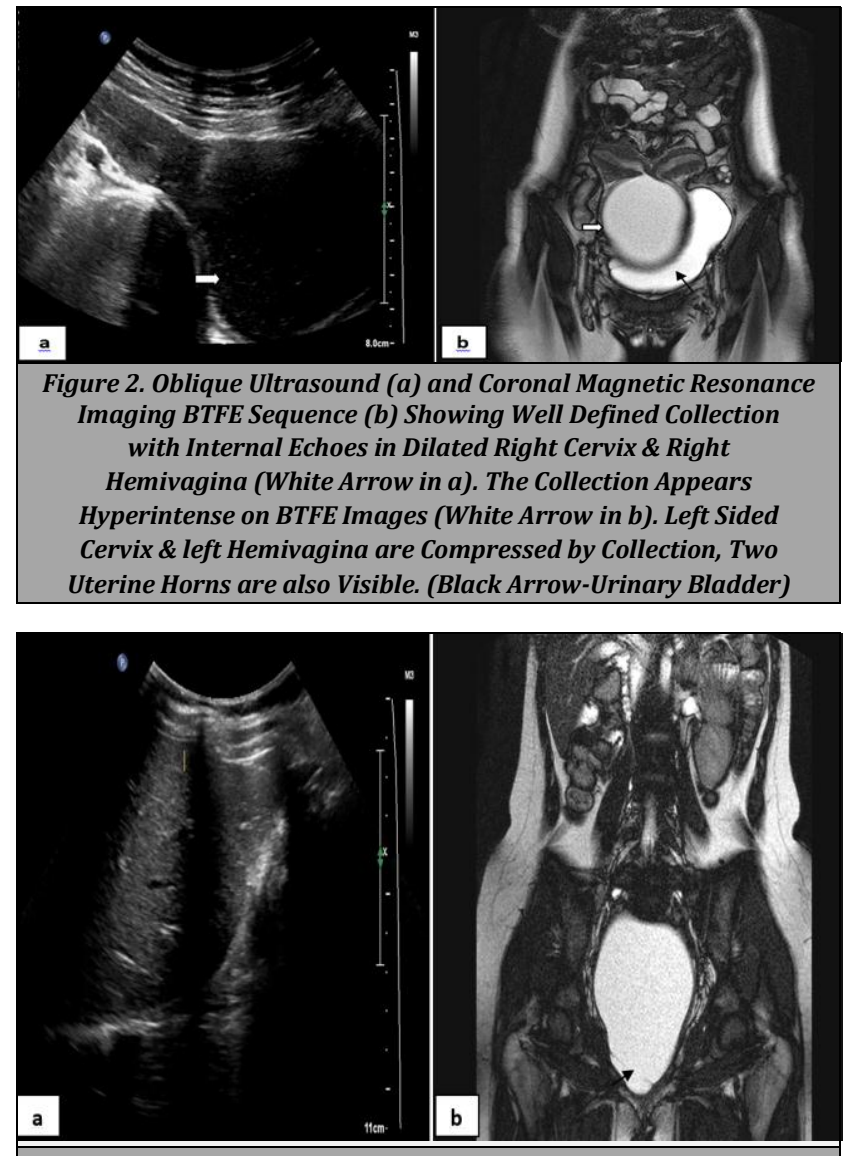

Figure 3. Oblique Ultrasound (a) and Axial Magnetic Resonance Imaging T1 Weighted (b) Showing Collection with Echoes Extending into the Lower Endometrial Cavity on the Right Side (Arrow in a). On T1 Weighted Imaging, Collection Extending into Lower Uterine Cavity Appears Hyperintense Indicating Blood Products / Haemorrhagic Contents (Arrow in b)

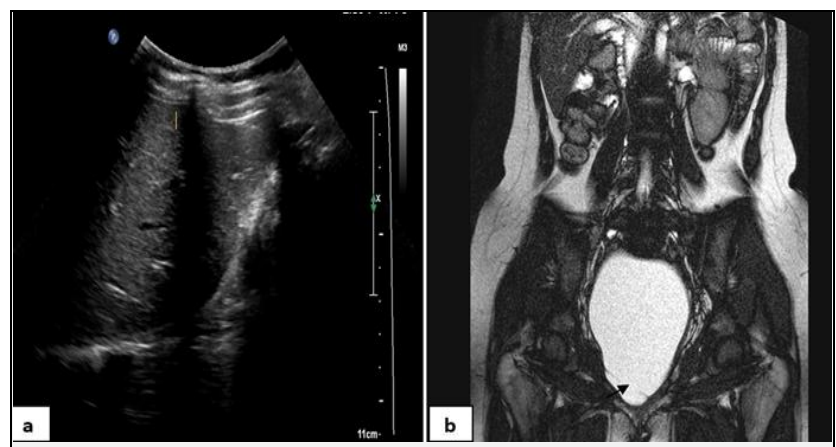

Figure 4. Sagittal Ultrasound (a) and Axial Magnetic Resonance Imaging T2 Weighted Showing Absent Right Kidney $(a, b)$ \& Normal Left Sided Kidney (b). Right Hemivagina is Markedly Distended with Hyperintense Collection (Blood Products / Haemorrhagic Contents). Transverse Septum is Seen in Its Inferior Aspect (Black Arrow in b)

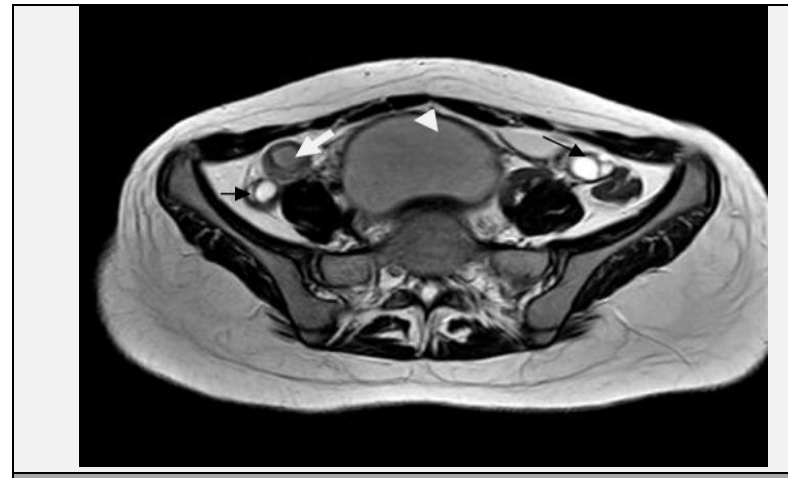

Figure 5. Axial Magnetic Resonance Imaging T2 Weighted Showing Hyperintense Collection Indicating Haemorrhagic / Blood Products. Also Seen in Dilated Right Fallopian Tube - Haematosalpinx (White Arrow) Signal Intensity is Similar to Collection in Right Hemivagina (White Arrowhead). Both Ovaries are also Visible (Black Arrows) which Appear Normal with Dominant Follicle on Left Side.

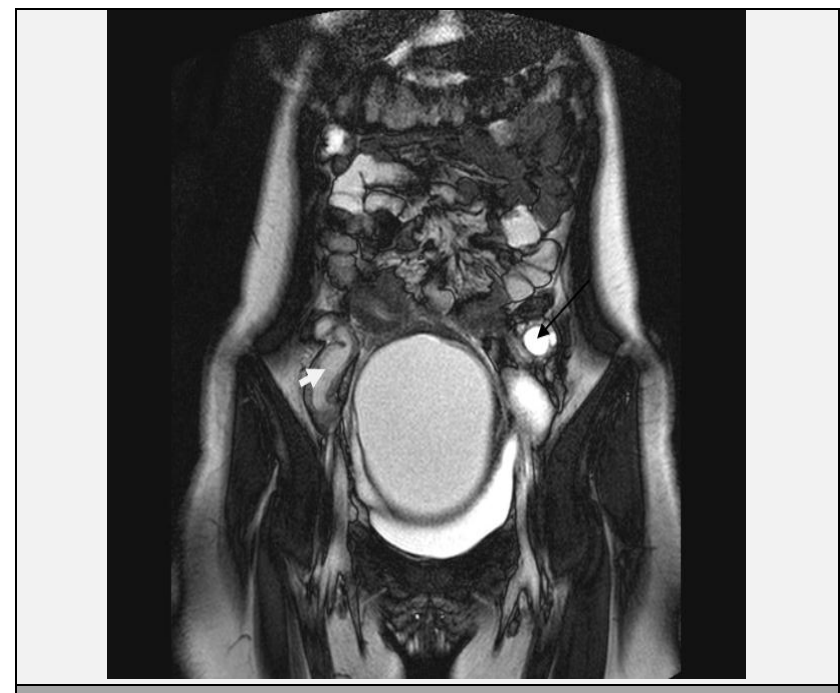

Figure 6. Coronal Magnetic Resonance Imaging BTFE Sequence Showing Dilated Tortuous Right Fallopian Tube with Hyperintense Collection within It - Haematosalpinx. Normal Left Ovary with Dominant Follicle is also Visualised (Black Arrow)

\section{DISCUSSION}

A failure of fusion of Mullerian ducts around approx. 6 to 12 weeks of gestational age leads to spectrum of Mullerian duct anomalies. $^{3}$ The pathogenesis is associated with a developmental anomaly of paramesonephric (Mullerian) and mesonephric (Wolffian) ducts. The mesonephric ducts induce the normal growth of paramesonephric ducts and helps in growth and development of both kidneys. So, any defect in growth and development of the mesonephric ducts leads to the unilateral renal agenesis and imperforated hemivagina accompanying OHVIRA syndrome.4 Fusion failure of the paramesonephric / Mullerian ducts leads to uterine didelphys and bicollis. Keeping in mind its association with a diversity of renal tract anomalies such as ectopic ureter, pre-operative renal tract imaging is done to restrain the probability of iatrogenic urinary incontinence. ${ }^{5}$ Most OHVIRA patients are diagnosed months after menarche mostly presented with haematocolpos, lower abdominal pain or pelvic pain, notable vaginal or pelvic mass, and occasionally together with abnormal vaginal discharge, fever, vomiting or acute retention of the urine. ${ }^{6}$ Delayed diagnosis can lead to more accumulation of blood products and eventually haematosalpinx as seen in 
our case study with worsening of reproductive outcome. Preconception counseling is mandatory for a high-risk case done during antenatal care period. Associated renal anomalies also increase the risk of preeclampsia / eclampsia during pregnancy and also influence the development of chronic renal and cardiovascular disease. ${ }^{7}$ First line of investigation for lower abdominal pain is usually ultrasonography, as it is fast, non-invasive \& easily accessible. However, MRI remains the imaging modality of choice to assess the Mullerian duct abnormalities. MRI will specifically depict uterine measurements like contour, shape, size, \& signal intensity with abnormalities of cervix and vagina. Pre-operative imaging with MRI is necessary to explain the Mullerian duct structures and renal tract precisely. MRI is also useful for planning surgeries. ${ }^{8}$ However, for the congenital gynaecological abnormalities laparoscopy is the gold standard for investigation but it is only useful if MRI is contraindicated or MRI fails, or MRI is unavailable. Treatment and management of OHVIRA is vaginal septum resection with the haemorrhagic fluid drainage from obstructed hemivagina and followed by vaginoplasty. It restores the vaginal function $\&$ also relieves the patient from recurrent urinary tract infection. However, postoperative complications like stenosis, reappearance of haematometra ${ }^{8}$ and infection are possibilities which require a second operation. Different treatment modalities have been tried to minimize the demand of second operation and to control the risk of post- operative re-obstruction such as the use of vaginal dilators and vaginal moulds ${ }^{9}$ and coated trachea-bronchial stent.

\section{CONCLUSIONS}

OHVIRA syndrome is an unusual cause of cyclic abdominal pain in prepubertal girl. It is associated with uterus didelphys (two horns), two cervices and two vagina, one of which is obstructed with ipsilateral renal anomaly. This syndrome is a little-known entity and can have atypical presentation, which results in difficulty in diagnosis and treatment delay. So proper radiological evaluation is needed for correct and early diagnosis. MRI plays a crucial role in the diagnosis as it is missed clinically. Early diagnosis with proper treatment also halts the adverse obstetric outcomes such as endometriosis, miscarriage, malpresentation, preterm birth, etc. \& it also preserves sexual / conception abilities. Surgery is done as a single stage / two stage procedure to excise / resect the septum \& relieve symptoms immediately. As concerned, all patients with ipsilateral renal agenesis mandatorily screened for Mullerian and duct anomalies and any pelvic mass in these patients should not be surgically treated immediately before proper radiological imaging.

Financial or other competing interests: None.

Disclosure forms provided by the authors are available with the full text of this article at jemds.com.

\section{REFERENCES}

[1] Dias JL, Jogo R. Herlyn-Werner-Wunderlich syndrome: pre-and post-surgical MRI and US findings. Abdom Imaging 2015;40(7):2667-82.

[2] Afrashtehfar CDM, Piña-García A, Afrashtehfar KI. Müllerian anomalies. Obstructed hemivagina and ipsilateral renal anomaly syndrome (OHVIRA). Cir Cir 2014;82(4):460-71.

[3] Buttram VC, Gibbons WE. Müllerian anomalies: a proposed classification (an analysis of 144 cases). Fertil Steril 1979;32(1):40-6.

[4] Ugurlucan FG, Bastu E, Gulsen G, et al. OHVIRA syndrome presenting with acute abdomen: a case report and review of the literature. Clin Imaging 2014;38(3):357-9.

[5] Schlomer B, Rodriguez E, Baskin L. Obstructed hemivagina and ipsilateral renal agenesis (OHVIRA) syndrome should be redefined as ipsilateral renal anomalies: cases of symptomatic atrophic and dysplastic kidney with ectopic ureter to obstructed hemivagina. J Pediatr Urol 2015;11(2):77-e1-6.

[6] Pansini L, Torricelli M, Gomarasca A, et al. Acute urinary retention due to didelphys uterus associated with an obstructed hemivagina in a 5-month-old infant. J Pediatr Surg 1988;23(10):984-5.

[7] Heinonen PK. Gestational hypertension and preeclampsia associated with unilateral renal agenesis in women with uterine malformations. Eur J Obstet Gynecol Reprod Biol 2004;114(1):39-43.

[8] Tanaka YO, Kurosaki Y, Kobayashi T, et al. Uterus didelphys associated with obstructed hemivagina and ipsilateral renal agenesis: MR findings in seven cases. Abdom Imaging 1998;23(4):437-41.

[9] Koyama-Sato M, Hashida O, Nakamura T, et al. Case of early postoperative adhesion in a patient with molimina due to transverse vaginal septum concomitant with imperforate hymen. J Obstet Gynaecol Res 2015;41(7):1141-4. 